\title{
Correlation of Electron Diffraction between t-EBSD in the SEM, CBED in the TEM and ACOM using ASTAR in the TEM using GaN Nanowires
}

Roy H. Geiss

Department of Chemistry, Colorado State University, Fort Collins, CO 80523-1872, USA

Transmission electron backscattered diffraction, t-EBSD, in the scanning electron microscope, SEM, was initially described in 2010 [1]. After slow initial acceptance, the number of publications in which it is used is increasing markedly. Many of the first applications took advantage of the higher resolution of t-EBSD in the study of thin films [2], [3]. More recently it is being used for pre-screening thin films and foils to determine which grains are best oriented to study certain defects in transmission electron microscopy, TEM [4], [5]. A major advantage of using t-EBSD in the SEM to screen thin films is that it is much easier and quicker to map the orientation of large areas of samples than with conventional diffraction techniques in the TEM. Automated crystal orientation mapping, ACOM, using precession electron diffraction, PED, in the TEM [6] offers an alternate approach to mapping the orientation of large sample areas without having to reposition the sample between the SEM and TEM.

In this work electron diffraction using t-EBSD in the SEM, convergent beam electron diffraction, CBED, in the TEM and ACOM in the TEM will be compared using data obtained from the same GaN nanowires applying the three techniques. The actual purpose of the study was to determine the growth properties of the nanowires, including the growth axis and fluctuations in orientation along that axis.

To facilitate locating the same nanowire in both the SEM and TEM for t-EBSD and CBED, a single tilt specimen holder for a JEM 2100F TEM was modified to be used in both the 2100F TEM and a JSM 6500F SEM with no repositioning of the sample. To ensure that the same nanowires were studied at AppFive, the GaN nanowires were dispersed on annotated carbon coated TEM grids. (ACOM analysis will be done on the same wires at the AppFive, LLC research facility in Tempe, AZ and be available for the meeting).

The t-EBSD patterns were collected in the JSM 6500F SEM using a EDAX Hikari Plus camera. The sample was tilted to -30 degrees with the stage tilted +60 degrees, Figure 1. In the JSM 6500F this allows the working distance, WD, to be varied from $5 \mathrm{~mm}$ up. The patterns obtained for this experiment were obtained at a $10 \mathrm{~mm}$ WD using $25 \mathrm{kV}$. Images were collected using either the SE detector in the SEM or a forward scattered detector, FSD, attached to the Hikari camera. Figure 2 shows an SE image of the area with the four GaN nanowires used in this study. The t-EBSD patterns were collected using line scans along the length of the wires; $10 \mathrm{~nm}$ steps were used resulting in collections of $200-400$ data points from a $2-4$ micron line scan. Images of the individual diffraction patterns from each point were collected as well as the Hough data. Individual patterns were indexed and the axial orientation of the wire was determined. It is assumed that the wires are lying flat on the carbon substrate, but this is not always the case. However, most lie within a few degrees of being flat so that the exact orientation of the nanowire axis can be determined to within a few degrees. Individual t-EBSD patterns from nanowires \#4 and \#7 clearly show that the axial orientations of the two nanowires are more than a few degrees different, Figures 3 and 6. Analysis software can be used ascertain point-to-point and point-to-origin 
misorientations of the patterns along a line scan of an individual wire. Figure 4 shows such a plot for wire \#4. In general an array of CBED diffraction patterns from an area in a TEM can only be obtained by manually moving the beam and saving the patterns. Also, in contrast to t-EBSD patterns, CBED patterns take considerably more time to analyze. Examples of a CBED pattern and a t-EBSD pattern from the same nanowire, \#7, are given in Figures 5 and 6, respectively. They show the same orientation, allowing for the approximately 45 degree $\mathrm{CCW}$ rotation between them.

The merits of collection and analysis of t-EBSD patterns in the SEM will be compared to the collection and analysis of both CBED patterns and ACOM - ASTAR data in the TEM.

\section{References:}

[1] RH Geiss, et al, Microsc Microanal 16(S2) (2010) p.1742-43.

[2] PW Trimby, et al, Ultramicroscopy 120(0) (2012) p.16-24.

[3] N Brodusch, et al, J Microscopy 250(1) (2013) p.1-14

[4] S. Suzuki, JOM, 65(9), (2013), p.1254-1263

[5] R. de Kloe, EDAX blog, October 2014

[6] D Viladot, et al, J Microscopy 252(1) (2013) p.23-34

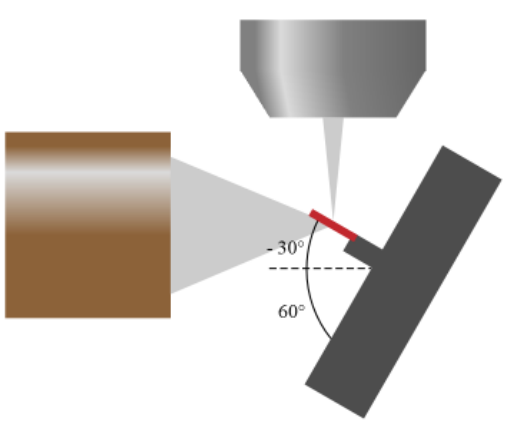

Figure 1. Experimental t-EBSD

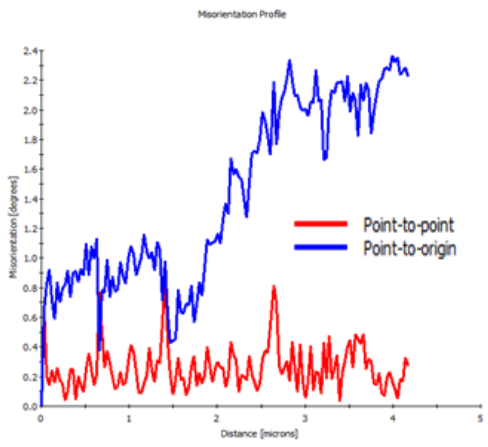

Figure 4. Misorientation plots

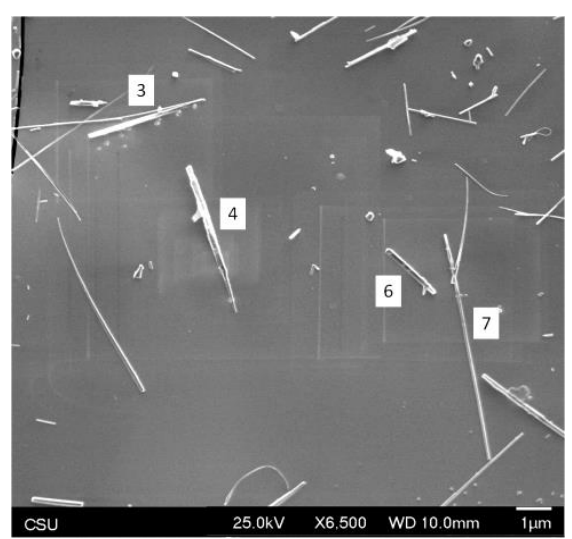

Figure 2. SE image of $\mathrm{GaN}$ wires

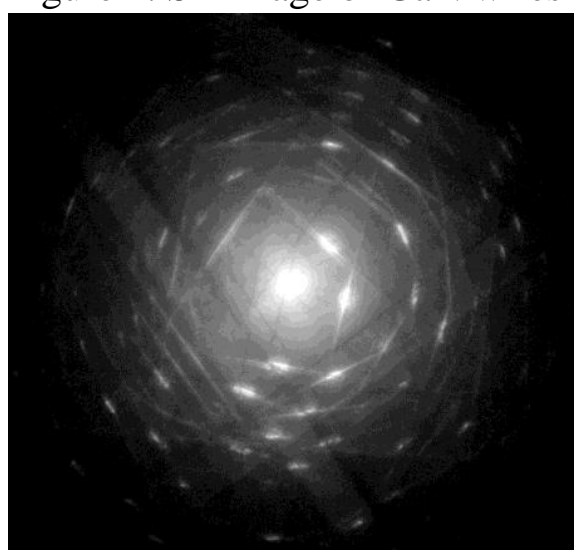

Figure 5. CBED from wire \#7

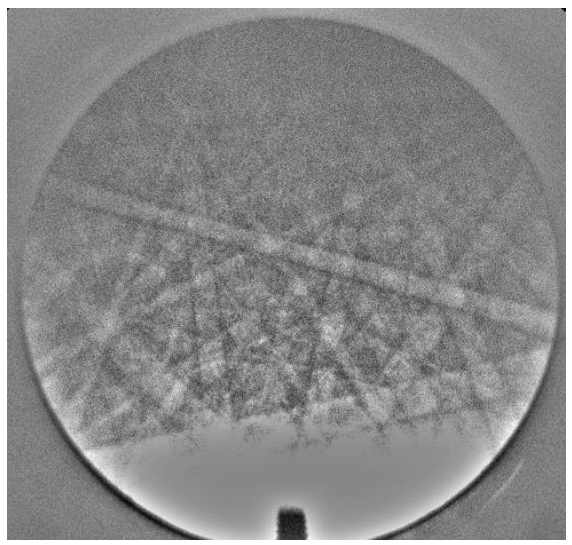

Figure 3. t-EBSD from wire \#4.

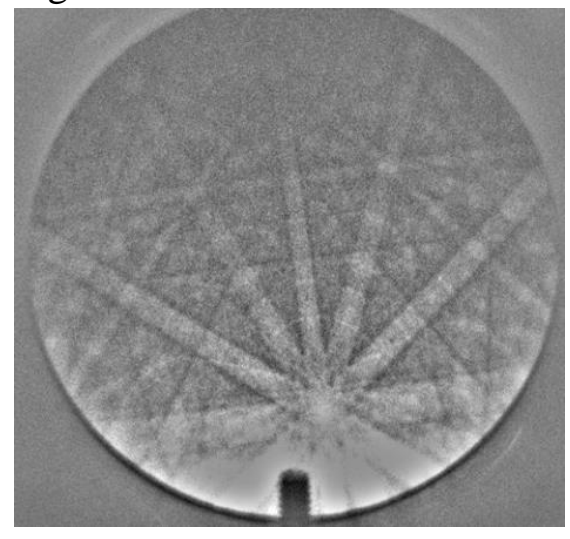

Figure 6. t-EBSD from wire \#7 